\title{
Light Exotic Isotopes : Recent Beam Developments and Physics Applications at ISOLDE
}

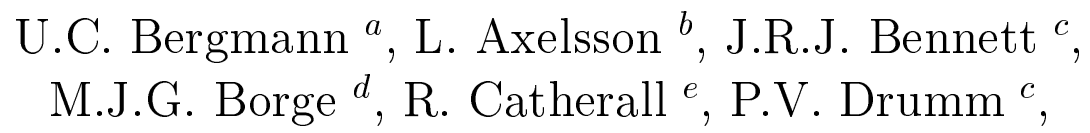
V.N. Fedoseyev ${ }^{f}$, C. Forssén ${ }^{b}$, L.M. Fraile ${ }^{d}$, H.O.U. Fynbo ${ }^{e}$, U. Georg ${ }^{e}$, T. Giles ${ }^{e}$, S. Grévy ${ }^{g}$, P. Hornsh $\varnothing \mathbf{j}{ }^{a}$, B. Jonson ${ }^{b}$, O.C. Jonsson ${ }^{e}$, U. Köster ${ }^{e}$, J. Lettry ${ }^{e}, \mathrm{~K}$. Markenroth ${ }^{b}$, F.M. Marqués ${ }^{g}$, V.I. Mishin ${ }^{f}$, I. Mukha ${ }^{h}$, T. Nilsson ${ }^{e}$, G. Nyman ${ }^{b}$, A. Oberstedt ${ }^{i}$, H.L. Ravn ${ }^{e}$, K. Riisager ${ }^{a}$, G. Schrieder ${ }^{h}$, V. Sebastian ${ }^{j}$, H. Simon ${ }^{e}$, O. Tengblad ${ }^{d}$, F. Wenander ${ }^{b}, \mathrm{~K}$. Wilhelmsen Rolander ${ }^{k}$

${ }^{a}$ Institut for Fysik og Astronomi, Aarhus Universitet, DK-8000 Aarhus C, Denmark

${ }^{b}$ Subatomär Fysik, Chalmers Tekniska Högskola, S-412 96 Göteborg, Sweden

${ }^{c}$ Rutherford Appleton Laboratory, CLRC, Chilton, Didcot, Oxon, OX11 0QX, UK

${ }^{d}$ Instituto Estructura de la Materia, CSIC, E-28006 Madrid, Spain

e EP/PS Divisions, CERN, CH-1211 Genève 23, Switzerland

$f$ Institute of Spectroscopy, Russian Academy of Sciences, RUS-142092 Troitsk, Russia

${ }^{g}$ Laboratoire de Physique Corpusculaire, IN2P3-CNRS, ISMRA et Université de Caen, F-14050 Caen Cedex, France

${ }^{h}$ Institut für Physik, Technische Hochschule, D-64289 Darmstadt, Germany

${ }^{i}$ Institutionen för Teknik och Naturvetenskap, Örebro Universitet, S-701 82 Örebro, Sweden

${ }^{j}$ Institut für Kernphysik, Johannes Gutenberg Universität, D-55099 Mainz, Germany

${ }^{k}$ Fysiska Institutionen, Stockholms Universitet, Box 6730, S-113 85 Stockholm, Sweden 
This paper is divided in three parts. (i) The measurement of yields and decay losses of $\mathrm{Li}$ and Be isotopes released from a thin foil tantalum target at the CERN/ISOLDE PS-Booster. (ii) Results from $\beta$-decay experiments on ${ }^{12} \mathrm{Be}$ and ${ }^{14} \mathrm{Be}$. An improved half-life of $21.49(3) \mathrm{ms}$ has been obtained for ${ }^{12} \mathrm{Be}$. (iii) The $\beta$-decay of ${ }^{9} \mathrm{C}$. An outline of the analysis procedure to determine the branching at high excitation energies is given. The ground-state branch has been determined to 54.1(15) \%. 
Experimental studies of nuclei far from stability often deal with low countrates since the production cross sections decrease rapidly towards the driplines. Thick targets used with the ISOL-method give high production rates but decay losses limit the yields of the many interesting nuclei which are short-lived. Thus, there is a request for targets with faster release properties.

One such target has recently been tested at ISOLDE. It consisted of 199 tantalum foils, $2 \mu \mathrm{m}$ thick, $1 \mathrm{~cm}$ high, and $15 \mathrm{~cm}$ long. Based on theoretical modelling the target mass and geometry were chosen to optimise the speed of diffusion and effusion processes within the foil structure [1]. Apart from the physics interest in improved beams of light neutron-rich nuclei these isotopes are also very suited for testing such a target since they often have very short half-lives. By bombarding the target with $\mu$ s pulses of $3 \cdot 10^{13}$ protons from the CERN/ISOLDE PS-Booster, 1 to 7 pulses per $14.4 \mathrm{~s}$ supercycle, the time structure of released $\mathrm{Li}, \mathrm{Na}$ and Be isotopes has been recorded. A tungsten surface ioniser was used in all tests. In addition, laser ionisation was applied for Be [2]. The data has been analysed in a diffusion-effusion model and physical parameters describing the release process have been extracted. The results are discussed in [3]. Here we will present the systematics, that is the variations in yields and decay losses over the isotope ranges of $\mathrm{Li}$ and $\mathrm{Be}$, from stability to the most neutron-rich and very short-lived ${ }^{11} \mathrm{Li}$ and ${ }^{14} \mathrm{Be}$.

Table 1 shows the yields, i.e. the number of ions extracted from the ion source per $\mu$ Coulomb of protons on target, measured either with a Faraday cup, via collection on a movable tape and subsequent $\beta$ measurement or via neutron detection in a $4 \pi$ neutron long-counter [4]. Note, that the target has demonstrated record yields of $7000(500){ }^{11} \mathrm{Li} / \mu \mathrm{C}, 48000(4000){ }^{12} \mathrm{Be} / \mu \mathrm{C}$ and $6.1(3.5)$ ${ }^{14} \mathrm{Be} / \mu \mathrm{C}$, the numbers in parenthesis being yields from standard tantalum-roll or uranium carbide targets. This was achieved with 5 to 10 times less target thickness. The tape station data of ${ }^{8} \mathrm{Li}$ and ${ }^{11} \mathrm{Be}$ were fitted with a parameterised release function $P(t)[5]$ and the released fraction, $R=\int P(t) e^{-\lambda t}$, was calculated for each radio-isotope with decay constant $\lambda$. The released fractions of ${ }^{9,11} \mathrm{Li}$ and ${ }^{12,14} \mathrm{Be}$ have improved by an order of magnitude compared to standard targets. The last two columns in Table 1 give the yields corrected for decay losses and the production rates calculated from Silberberg and Tsao (S\&T) [6] cross sections. The ratio of these two numbers should equal the ionisation efficiency which has been measured off-line to be 0.9 for $\mathrm{Li}$ and 0.07 for Be [7]. The S\&T cross sections fail in some specific cases but can reproduce the overall trends. 
We have initiated a detailed experimental program on the $\beta$-decay of the heaviest Be isotopes, taking advantage of the improved Be yields described above. One result was an improved half-life of ${ }^{12} \mathrm{Be}$. Fig. 1 shows the measured time distributions of $\beta$ particles and delayed neutrons from ${ }^{12} \mathrm{Be}$, giving a weighted mean value of $\mathrm{T}_{1 / 2}=21.49(3) \mathrm{ms}$. The small uncertainty, obtained in the fit of the $\beta$ spectrum, relies on the good precision of the ${ }^{12} \mathrm{~B}$ half-life, $\mathrm{T}_{1 / 2}=$ $20.20(2) \mathrm{ms}$ [8]. The literature value of the ${ }^{12} \mathrm{Be}$ half-life is $21.3(1) \mathrm{ms}$. New results on the one-, two- and three-neutron branches from ${ }^{12} \mathrm{Be}$ and ${ }^{14} \mathrm{Be}$ have already been reported [4]. It was concluded that ${ }^{14} \mathrm{Be}$ essentially only decays via one-neutron emission and strict upper limits were put on the multi-neutron branches as well as the $\beta$ feeding of particle bound states in ${ }^{14} \mathrm{~B}$. Recently, using a combined setup of a ${ }^{3}$ He spectrometer and a gas-Si telescope, we have measured the neutron energy distribution and $\beta$-delayed charged particles from ${ }^{14} \mathrm{Be}$. We confirm the existence of a strongly fed neutron group at $287 \mathrm{keV}$ as found in [9]. No indication was found for neutrons below $200 \mathrm{keV}$ and upper limits of $8.4 \%$ and $17.6 \%$, one- and two-sigma confidence levels, can be assigned to the feeding of neutrons from $30 \mathrm{keV}$ to $200 \mathrm{keV}$. Thus, the Gamov-Teller strength to the lower part of excitation energies is dominated by one strong transition to a state $287 \mathrm{keV}$ above the one-n threshold. The data on charged particles indicate a branch, on the per mille level, of alphas below $1 \mathrm{MeV}$ but a dedicated experiment is needed to give final proof. It would give an interesting similarity to the decay of ${ }^{9,11} \mathrm{Li}$ and ${ }^{6,8} \mathrm{He}$ with a strong Gamov-Teller transition to a state in ${ }^{14} \mathrm{~B}$ roughly $3 \mathrm{MeV}$ below the decaying ground state. The spectrum of ${ }^{12}$ Be neutrons was also recorded and is essentially uniform below $1300 \mathrm{keV}$ with a slight grouping seen around 200 and $900 \mathrm{keV}$.

\section{Beta-decay of ${ }^{9} \mathrm{C}$}

Experiments have indicated an unusually large $\beta$-strength asymmetry in the

mirror decays ${ }^{9} \mathrm{C} \stackrel{\beta}{\rightarrow}{ }^{9} \mathrm{~B}$ and ${ }^{9} \mathrm{Li} \stackrel{\beta}{\rightarrow}{ }^{9} \mathrm{Be}$, most pronounced in the feeding of a highly excited state around $12 \mathrm{MeV}[10,11]$. To clear up uncertainties in the ${ }^{9} \mathrm{C}$ decay two experiments have been performed at ISOLDE. One was aiming at a more accurate determination of the ground-state branch. According to literature this branch is 60(10) \%, determined via beam monitoring [10]. Since ${ }^{9} \mathrm{~B}$ is unbound and so are the ${ }^{8} \mathrm{Be}$ and ${ }^{5} \mathrm{Li}$ subsystems the ${ }^{9} \mathrm{C}$ decay always proceeds to a final state of $\alpha+\alpha+$ p. Our experiment involved a direct measurement of $164 \mathrm{keV}$ protons with a $25 \mu \mathrm{m}$ silicon detector, resulting in a ground-state branch of 54.1(15) \%. The data are shown in Fig. 2. The result 
was obtained by normalising the proton peak to the remaining counts at higher energies, knowing that the two alphas from the ground-state transition had energies below our threshold. As seen in Fig. 2 a clear separation was obtained of the proton peak from noise and $\beta$ signals. Only protons with energies above $10 \mathrm{MeV}$ gave energy losses below $200 \mathrm{keV}$ and this rather small component was subtracted using coincident data from a thick silicon back detector.

Below, the analysis of the second experiment is discussed. A complete description of the analysis procedure and the full set of results will be given in a later publication [12]. The setup was optimised to determine the feeding at high excitation energies. A beam of $800{ }^{9} \mathrm{C}^{16} \mathrm{O}^{-}$ions/s was deposited in a thin carbon foil situated between two parallel 16x16 segmented double sided silicon strip detectors. From these data the width of the $12 \mathrm{MeV}$ state was determined as $450 \mathrm{keV}$. The feeding of the $12 \mathrm{MeV}$ state can be determined if its break-up via the ground state of ${ }^{8} \mathrm{Be}$ is known relative to all its other break-up channels. This is possible since the sequential break-up via the ${ }^{8} \mathrm{Be}$ ground state gives a proton peak at $10.8 \mathrm{MeV}$ of width $400 \mathrm{keV}$ which can be normalised to all ${ }^{9} \mathrm{C}$ decays. The normalisation relies on the knowledge of the ${ }^{9} \mathrm{~B}$ ground-state branch, since the $164 \mathrm{keV}$ protons are not seen with this setup due to $\beta$-contamination.

For the purpose of finding the relative branching of the $12 \mathrm{MeV}$ state extensive Monte-Carlo simulations have been developed assuming break-up via ${ }^{8} \mathrm{Be}+\mathrm{p}$ or ${ }^{5} \mathrm{Li}+\alpha$ ground or first excited states. Simulations are needed to include kinematic effects since the four channels have quite different geometrical acceptances for three-particle detection. The setup determines the energies and directions of all detected particles, and for three-particle coincidences mass identification can also be obtained as demonstrated in Fig. 3. Knowing which particle is the proton facilitates the determination of the relative weight of the channels since the comparison between simulation and data can be based on more information.

\section{References}

[1] J.R.J. Bennett et al., Nucl. Instr. and Meth. in Phys. Res. B155 (1999) 515.

[2] V.I. Mishin et al., Nucl. Instr. and Meth. in Phys. Res. B73 (1993) 550.

[3] "Release Studies of a Thin Foil Tantalum Target for the Production of Shortlived Radioactive Nuclei". J.R.J. Bennett et al., these proceedings.

[4] U.C. Bergmann et al., Nucl. Phys. A658 (1999) 129.

[5] J. Lettry et al., Nucl. Instr. and Meth. in Phys. Res. B126 (1997) 130. 
[6] R. Silberberg and C.H. Tsao, Astrophys. J. Suppl. Series No. 220(II) 25 (1973) 335.

[7] U. Köster et al., p. 989 in Proc. from ENAM98, Exotic Nuclei and Atomic Masses (Bellaire, 1998), eds B.M. Sherrill, D.J. Morrissey and C.N. Davids (AIP Conf. Proc. 455, 1998).

[8] G. Audi et al., Nucl. Phys. A624 (1997) 1.

[9] M.D. Belbot et al., Phys. Rev. C56 (1997) 3038.

[10] D. Mikolas et al., Phys. Rev. C37 (1988) 766.

[11] G. Nyman et al., Nucl. Phys. A510 (1990) 189.

[12] U.C. Bergmann et al., in preparation, to be submitted to Nucl. Phys. A. 
Table 1

Measured yields and released fractions $(\mathrm{R})$ of lithium and beryllium isotopes from a $2 \mu \mathrm{m}$ tantalum foil target. The measuring device used was either a Faraday cup (F), a tape station ( $\mathrm{T}$ ) associated with a $\beta$ counter, or a neutron counter $(\mathrm{N})$. The change in production cross section per unit mass step when moving towards the neutron drip-line was calculated for each isotope taking the ratio of yield/ $\mathrm{R}$ to its value for the preceding isotope. The yield corrected for decay losses can be compared with the production rate calculated with Silberberg and Tsao (S\&T) [6] cross sections.

\begin{tabular}{ccccccc}
\hline Ion & $\begin{array}{c}\mathrm{T}_{1 / 2} \\
(\text { from }[8])\end{array}$ & $\begin{array}{c}\text { Yield } \\
(1 / \mu \mathrm{C})\end{array}$ & $\begin{array}{c}\mathrm{R} \\
(\%)\end{array}$ & $\begin{array}{c}\sigma \text { ratio } \\
\mathrm{A} /(\mathrm{A}-1)\end{array}$ & $\begin{array}{c}\text { Yield } / \mathrm{R} \\
(1 / \mu \mathrm{C})\end{array}$ & $\begin{array}{c}\text { S\&T prod. } \\
\text { rate }(1 / \mu \mathrm{C})\end{array}$ \\
\hline${ }^{7} \mathrm{Li}(\mathrm{F})$ & stable & $2.0 \cdot 10^{9}$ & & & $2.0 \cdot 10^{9}$ & $2.2 \cdot 10^{9}$ \\
${ }^{8} \mathrm{Li}(\mathrm{T})$ & $838(6) \mathrm{ms}$ & $5.8 \cdot 10^{8}$ & 75 & $1 / 2.6$ & $7.7 \cdot 10^{8}$ & $4.2 \cdot 10^{8}$ \\
${ }^{9} \mathrm{Li}(\mathrm{T})$ & $178.3(4) \mathrm{ms}$ & $1.7 \cdot 10^{7}$ & 45 & $1 / 20$ & $3.8 \cdot 10^{7}$ & $3.2 \cdot 10^{8}$ \\
${ }^{11} \mathrm{Li}(\mathrm{N})$ & $8.59(14) \mathrm{ms}$ & $7.0 \cdot 10^{3}$ & 2 & $1 / 10$ & $3.5 \cdot 10^{5}$ & $5.1 \cdot 10^{5}$ \\
${ }^{9} \mathrm{Be}(\mathrm{F})$ & stable & $6.3 \cdot 10^{8}$ & & & $6.3 \cdot 10^{8}$ & $3.5 \cdot 10^{8}$ \\
${ }^{10} \mathrm{Be}(\mathrm{F})$ & $1.51(6) \mathrm{My}$ & $4.9 \cdot 10^{8}$ & & $1 / 1.3$ & $4.9 \cdot 10^{8}$ & $3.1 \cdot 10^{8}$ \\
${ }^{11} \mathrm{Be}(\mathrm{T})$ & $13.81(8) \mathrm{s}$ & $3.4 \cdot 10^{6}$ & 82 & $1 / 100$ & $4.1 \cdot 10^{6}$ & $5.8 \cdot 10^{7}$ \\
${ }^{12} \mathrm{Be}(\mathrm{N})$ & $21.3(1) \mathrm{ms}$ & $4.8 \cdot 10^{4}$ & 1.8 & $1 / 1.5$ & $2.7 \cdot 10^{6}$ & $7.3 \cdot 10^{6}$ \\
${ }^{14} \mathrm{Be}(\mathrm{N})$ & $4.35(17) \mathrm{ms}$ & 6.1 & 0.05 & $1 / 15$ & $1.2 \cdot 10^{4}$ & $1.6 \cdot 10^{4}$ \\
\hline
\end{tabular}




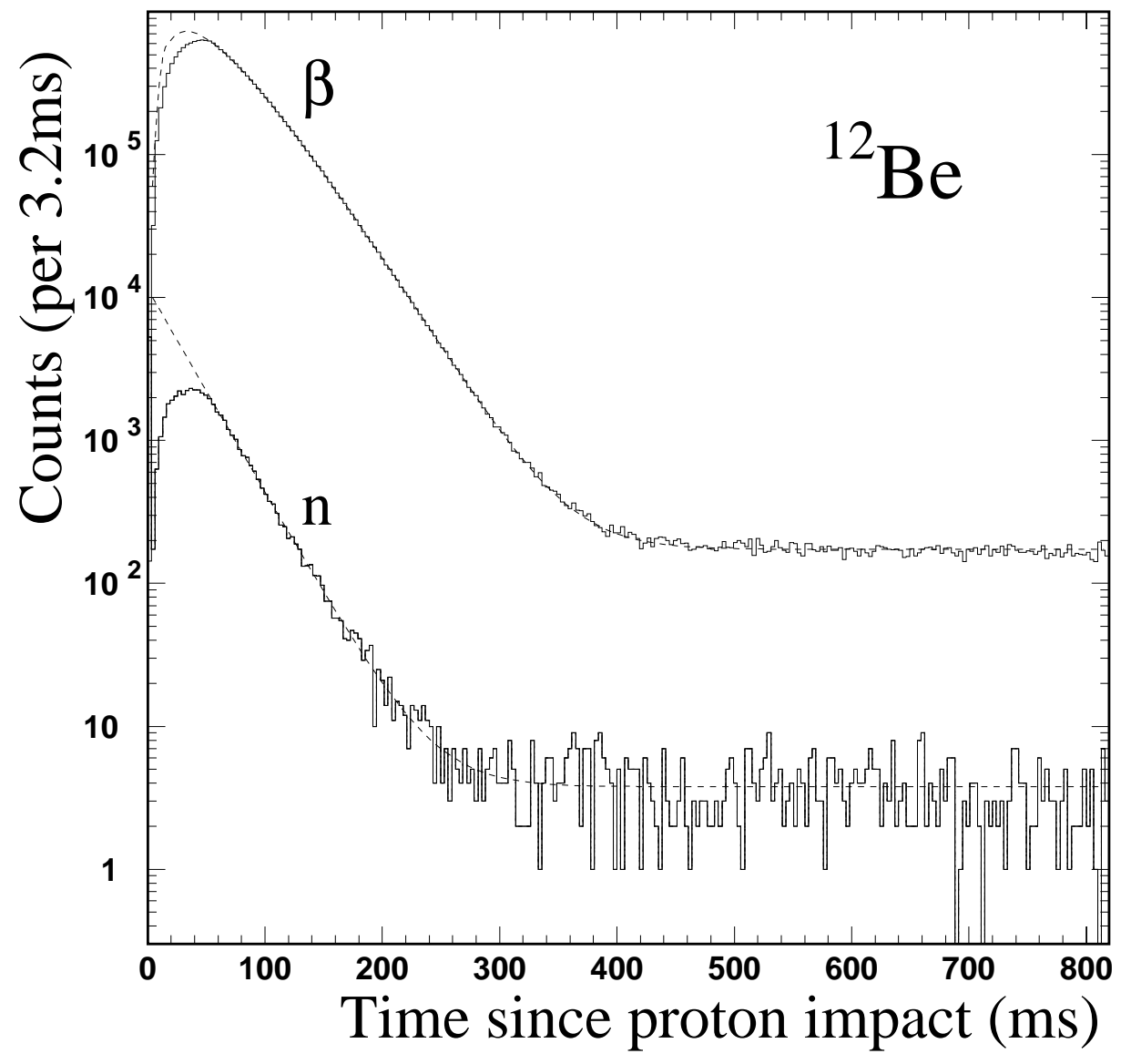

Fig. 1. Detection times of neutrons and $\beta$ particles from the decay of ${ }^{12} \mathrm{Be}$. The beam was shut off by an electrostatic beam gate at $53 \mathrm{~ms}$ after proton impact. From this starting point the neutron decay was fitted with an exponential+background whereas the $\beta$ spectrum was described as a chain decay of ${ }^{12} \mathrm{Be}$ and its daughter ${ }^{12} \mathrm{~B}$, assuming initial amounts of both nuclei. 

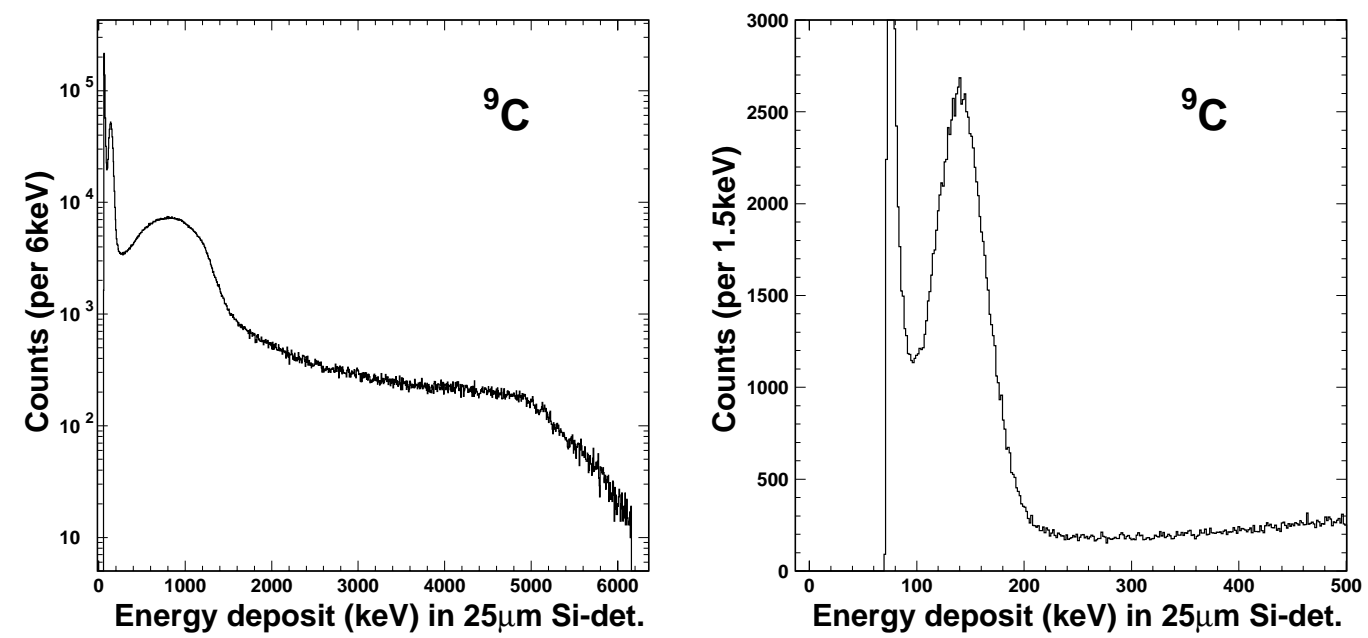

Fig. 2. Energy deposit in a $25 \mu \mathrm{m}$ silicon detector of protons and alphas emitted in the decay of ${ }^{9} \mathrm{C}$. On the right the region around the $164 \mathrm{keV}$ proton peak has been expanded. 


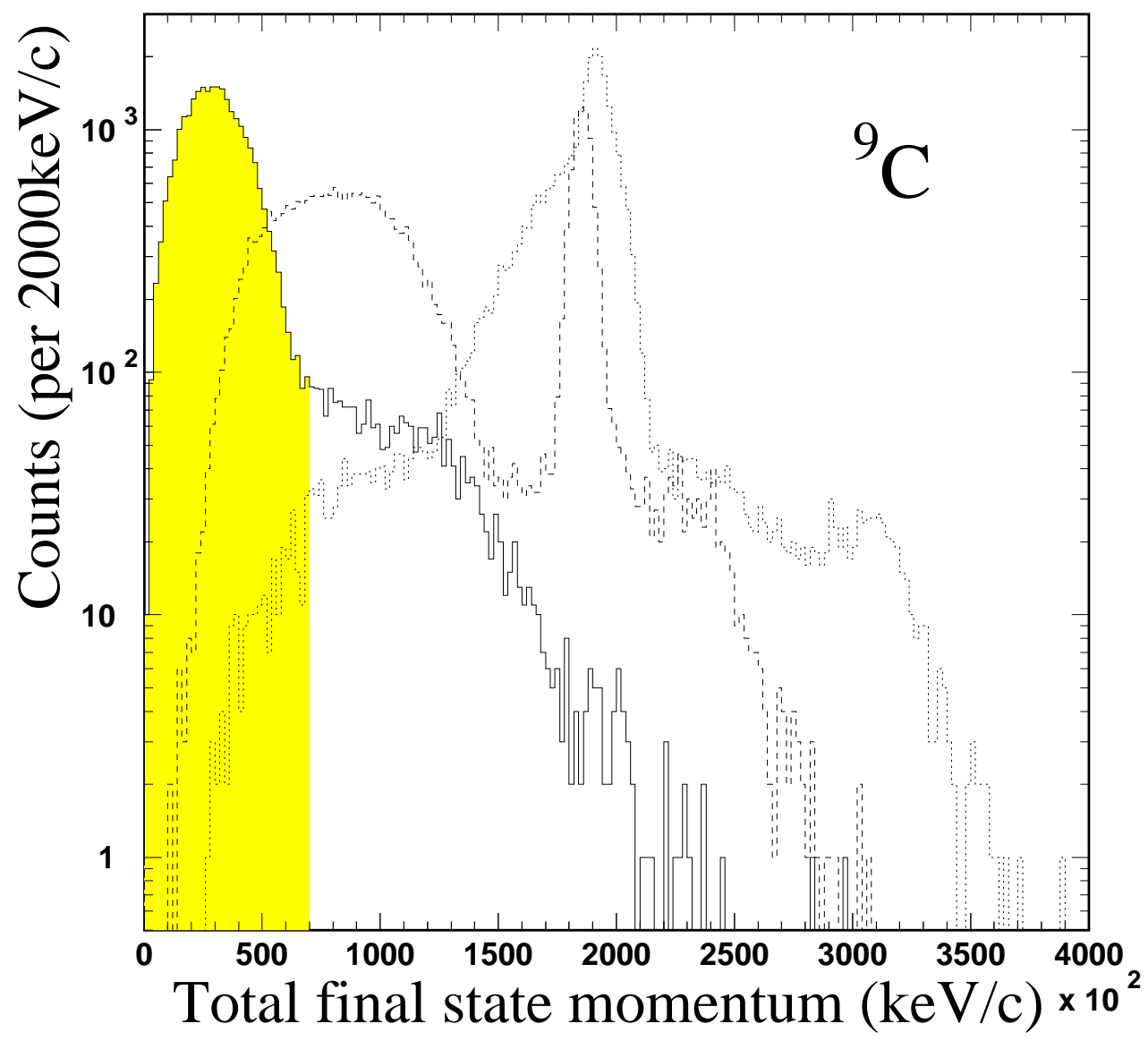

Fig. 3. To find out which particle is the proton in a three-particle event of the ${ }^{9} \mathrm{C}$ data the total momentum is calculated for the three possibilities. The solid, dashed and dotted histograms are the resulting lowest, second lowest and highest momenta in the break-up of the $12 \mathrm{MeV}$ state. Only events in the filled area are accepted to reduce $\beta$-contamination. 